AZEVEDO AM; ANDRADE JÚNIOR VC; PEDROSA CE; VALADARES NR; ANDRADE RF; SOUZA JRS. 2016. Estudo da repetibilidade genética em clones de couve. Horticultura Brasileira 34: 054-058. DOI - http://dx.doi.org/10.1590/S0102-053620160000100008

\title{
Estudo da repetibilidade genética em clones de couve
}

\author{
Alcinei M Azevedo'; Valter C Andrade Júnior²; Carlos E Pedrosa²; Nermy R Valadares²; Renner F \\ Andrade² Jéssyca RS Souza \\ ${ }^{1}$ UFV, Viçosa-MG, Brasil; alcineimistico@hotmail.com (autor para correspondência); ${ }^{2}$ UFVJM, Diamantina-MG, Brasil; valterjr@ \\ ufvjm.edu.br; carlosenrrik@yahoo.com.br; nermyvaladares@hotmail.com; rennerandrade@hotmail.com; jessycasilva123@hotmail.com
}

\section{RESUMO}

Durante o ciclo vegetativo da couve é possível fazer várias colheitas. Dessa forma, é necessário estabelecer o número mínimo de colheitas que possibilita a seleção confiável de genótipos superiores. Objetivou-se estimar o número mínimo de medições para a seleção de clones de couve com maior eficiência e confiabilidade por meio do estudo de repetibilidade. O experimento foi conduzido na Universidade Federal dos Vales do Jequitinhonha e Mucuri, utilizando o delineamento em blocos casualizados com 27 genótipos de couve, quatro repetições e cinco indivíduos por parcela. Durante 10 semanas foram avaliadas semanalmente a altura das plantas, diâmetro do caule, comprimento e largura do limbo foliar, número de folhas comerciais, número de folhas totais e número de brotações. Foram utilizados os métodos da análise de variância, componentes principais e análise estrutural no estudo de repetibilidade. As características estudadas tiveram altas estimativas do coeficiente de repetibilidade. Apenas três colheitas possibilitam a seleção confiável dos melhores genótipos para todas as características avaliadas.

Palavras-chave: Brassica oleracea var. acephala, número de avaliações, número de medições, análise multivariada.

\section{ABSTRACT}

\section{Study of genetic repeatability in kale clones}

During the growing season of kale, several harvests may be carried out. Thus, establishing the minimum number of measurements for a reliable selection of superior genotypes is necessary. This research aimed to estimate the minimum number of measurements for selecting kale clones with greater efficiency and reliability through the repeatability study. The experiment was carried out at Universidade Federal dos Vales do Jequitinhonha e Mucuri using randomized block design with 27 genotypes of kale, four replications and five individuals per plot. During 10 weeks we evaluated weekly the following parameters: plant height, stem diameter, length and width of the leaf blade, number of marketable leaves, number of total leaves and number of shoots. We used analysis of variance methods, principal components analysis and structural analysis in study of repeatability. The characteristics studied showed high values for estimating the coefficient of repeatability. Only three harvests are sufficient to permit reliable selection of the best genotypes for all traits.

Keywords: Brassica oleracea var. acephala, number of evaluations, number of measurements, multivariate analysis.

\section{(Recebido para publicação em 29 de maio de 2014; aceito em 12 de agosto de 2015)} (Received on May 29, 2014; accepted on August 12, 2015)

\begin{abstract}
A couve (Brassica oleracea var. acephala), hortaliça arbustiva considerada anual ou bienal, é uma das variedades mais antigas da família Brassicaceae, originada no leste do mediterrâneo e utilizada para a alimentação humana há mais de 2.000 anos (Balkaya \& Yanmaz, 2005), cujo consumo tem aumentado gradativamente no Brasil (Novo et al., 2010). Bem como as outras brássicas, esta cultura apresenta alto teor de água e baixo teor de lipídeos, carboidratos e propriedades calóricas (Vilar et $a l ., 2008$ ), além de apresentar ação anticarcinogênica, uma vez que é fonte rica de glucosinolatos e possui elevado teor de flavonóides, vitaminas e nutrientes
\end{abstract}

minerais (Moreno et al., 2006).

Segundo Azevedo et al. (2012), é necessário um intenso trabalho de melhoramento para esta cultura, principalmente para aumentar sua produtividade de folhas com padrão comercial e resistência ao ataque de Brevicoryne brassicae e Plutella xylostella, principais pragas dessa hortaliça. Além do melhoramento para resistência a pragas, estes mesmos autores consideram a necessidade de selecionar genótipos com menor altura, menor número de brotações e maior número de folhas comerciais, a fim de facilitar os tratos culturais e aumentar a produção por área.

Durante o processo de seleção de plantas com características desejáveis, visando a recomendação de cultivares ou a escolha de genótipos superiores nos programas de melhoramento, é importante certificar-se de sua superioridade genética. Desta forma, a análise de sucessivas medições de uma característica em um grupo de indivíduos é desejável no melhoramento genético de plantas, pois é esperado que a superioridade ou a inferioridade inicial de um indivíduo se mantenha ao longo das medições (Martuscello et al., 2007).

No processo de seleção de genótipos superiores de couve, é necessária a realização de avaliações periódicas em cada indivíduo sob seleção, uma vez que 
são feitas múltiplas colheitas no ciclo da planta, resultando em um emprego considerável de tempo e custo. De acordo com Neves et al. (2010), a veracidade do bom desempenho de um genótipo ao longo de sucessivas avaliações pode ser comprovada pelo coeficiente de repetibilidade das características estudadas nos programas de melhoramento.

O coeficiente de repetibilidade varia com as propriedades genéticas da população (por exemplo: clones, progênies de meios-irmãos e progênies de irmãos completos) e com a natureza do caráter avaliado. Suas estimativas variam de 0,00 a 1,00, sendo 1,00 a repetibilidade máxima, verificada quando uma das características manifesta-se com muita constância (Cruz et al., 2012). Outra importante informação do estudo de repetibilidade é a predição do número de medições necessárias para obter um determinado nível de precisão na comparação entre genótipos para uma dada característica. Tal informação possibilita a avaliação de um número mínimo de medições, reduzindo o gasto de tempo, mão de obra e custo. Embora tais estudos sejam muito importantes, poucas são as informações sobre a avaliação de genótipos de couve encontradas na literatura.

Dentre os métodos estatísticos possíveis no estudo da repetibilidade, os mais utilizados são o método da análise de variância, o método dos componentes principais baseando-se em matrizes de covariância e correlações obtidas dos dados dos clones para cada par de épocas de avaliação e analise estrutural (Cruz et al., 2012). Desta forma, objetivou-se estimar o número mínimo de medições para a seleção de clones de couve com maior eficiência e confiabilidade por meio do estudo de repetibilidade.

\section{MATERIAL E MÉTODOS}

O experimento foi conduzido no Setor de Olericultura, localizado no campus JK da UFVJM, Diamantina-MG (18 $8^{\circ} 12^{\prime} 01^{\prime \prime} \mathrm{S}$; 4334'20' O, altitude $1.387 \mathrm{~m})$. No período de condução do experimento, a temperatura média foi de $18,3^{\circ} \mathrm{C}$ com umidade relativa do ar de $80,3 \%$.

Foram avaliados vinte e sete genótipos de couve do banco de germoplasma da UFVJM utilizando-se o delineamento em blocos casualizados com quatro repetições e cinco plantas por parcela, sendo dezenove genótipos oriundos de coletas em propriedades rurais na região de Diamantina-MG (UFVJM-2, UFVJM-3, UFVJM-4, UFVJM-5, UFVJM-7, UFVJM-8, UFVJM-9, UFVJM-10, UFVJM-13, UFVJM-19, UFVJM-21, UFVJM-22, UFVJM-24, UFVJM-26, UFVJM-27, UFVJM-30, UFVJM-32, UFVJM-34 e UFVJM-36) e oito genótipos coletados em propriedades rurais na região de Lavras-MG doados pela Universidade Federal de Lavras (UFLA-1, UFLA-3, UFLA-6, UFLA-8, UFLA-5, UFLA-10, UFLA-11 e UFLA-12).

Para a instalação do experimento as brotações foram coletadas em 28 de julho de 2011 e usadas para formação das mudas pelo sistema de estaquia verde, coletando-se as brotações laterais com três a quatro centímetros de altura e dois folíolos, no terço basal das plantas matrizes. Após a coleta, os brotos foram plantados em bandejas de isopor de 72 células preenchidas com substrato comercial Plantimax $^{\circledR}$ e mantidas em casa de vegetação no Setor de Olericultura da UFVJM por 30 dias, a fim de garantir o melhor enraizamento das brotações. Para o transplantio das mudas foram utilizados vasos com capacidade de 7,5 $\mathrm{kg}$ de solo preenchidos com latossolo vermelho distrófico e esterco bovino na proporção 3:1. Para garantir o melhor pegamento das mudas, os vasos foram mantidos em casa de vegetação por 30 dias. Em 28 de setembro de 2011 os vasos foram levados para o campo, onde permaneceram até a coleta dos dados para a avaliação agronômica. As adubações de solo e cobertura foram realizadas de acordo com as recomendações para a cultura (Filgueira, 2008) e o controle de pulgões e lagartas foi feito manualmente em dias alternados. Semanalmente, foram retiradas as folhas senescentes e as brotações para possibilitar o melhor desenvolvimento das plantas.

A partir de 2 de novembro de 2011, durante 10 semanas, avaliou-se sema- nalmente em cada indivíduo a altura das plantas utilizando fita métrica graduada em cm (a partir do nível do solo até a extremidade das folhas mais altas), o diâmetro do caule em mm (medido com paquímetro na metade da altura da planta), o comprimento e largura do limbo foliar utilizando régua graduada $\mathrm{em} \mathrm{cm}$ (selecionando-se a $5^{\mathrm{a}}$ folha expandida mais nova), o número de folhas apropriadas para a comercialização (obtida pela contagem das folhas maiores que $15 \mathrm{~cm}$ e sem sinais de senescência), o número total de folhas na planta (obtida pela contagem de todas as folhas desconsiderando as não expandidas e as folhas existentes nas brotações) e o número de brotações.

Para o estudo de repetibilidade utilizou-se o modelo estatístico com dois fatores de variação (medição e genótipo) conforme sugerem Cruz et al. (2012) para experimentos similares a este. $\mathrm{Y}_{\mathrm{ij}}=$ $\mathrm{m}+\mathrm{g}_{\mathrm{i}}+\mathrm{a}_{\mathrm{j}}+\mathrm{e}_{\mathrm{ij}}$, sendo: $\mathrm{Y}_{\mathrm{ij}}$ : observação referente ao i-ésimo genótipo na j-ésima medição (avaliação); m: média geral; $g_{i}$ : efeito aleatório do i-ésimo genótipo sob a influência do ambiente permanente (i $=1,2, \ldots, 27$ genótipos); $\mathrm{a}_{\mathrm{j}}$ : efeito $\mathrm{da}$ j-ésima medição $(j=1,2, \ldots, 10)$ e, $e_{i j}$ : erro experimental associado à observação $\mathrm{Y}_{\mathrm{ij}}$.

Para atender as pressuposições da análise de variância, os dados obtidos por contagem foram submetidos à transformação em raiz quadrada. Para a estimativa dos coeficientes de repetibilidade, do número mínimo de medições e do coeficiente de determinação, utilizaram-se os métodos: análise de variância (ANOVA); componentes principais com base na matriz de correlações e de covariâncias; e análise estrutural com base na matriz de correlações. Toda a análise estatística foi realizada utilizando o software genético-estatístico GENES (Cruz, 2013) e todas as expressões estatísticas utilizadas são detalhadas por Cruz et al. (2012).

\section{RESULTADOS E DISCUSSÃO}

Verificou-se diferença para todas as características em nível de $1 \%$ de probabilidade pelo teste $\mathrm{F}$ entre os genótipos de couve (Tabela 1). Esses 
Tabela 1. Resumo da análise de variância dos caracteres altura da planta, diâmetro do caule, comprimento do limbo foliar, largura do limbo foliar, número de folhas comerciais, número de folhas totais e número de brotações em 10 medições, em 27 clones de couve (summary of variance analysis of characters plant height, stem diameter, length and width of leaf blade, number of marketable and total leaves and number of shoots on 10 measurements of 27 kale clones). Diamantina, UFVJM, 2012.

\begin{tabular}{lccccccc}
\hline FV & AP & DC & CF & LF & NFC & NFT & NB \\
\hline Medições & 2128,056 & 96,601 & 123,198 & 79,246 & 3,232 & 1,112 & 3,433 \\
Genótipos & $2541,009^{* *}$ & $66,751^{* *}$ & $195,853^{* *}$ & $105,312^{* *}$ & $1,553^{* *}$ & $11,934^{* *}$ & $3,719^{* *}$ \\
Resíduo & 15,076 & 0,731 & 3,937 & 0,968 & 0,031 & 0,027 & 0,068 \\
\hline CV (\%) & 9,343 & 12,474 & 17,191 & 11,293 & 16,244 & 5,410 & 15,571 \\
\hline
\end{tabular}

${ }^{* *}$ significativo pelo teste $\mathrm{F}$ ao nível de 1\%. Altura da planta (AP), diâmetro do caule (DC), comprimento da folha (CF), largura da folha (LF), número de folhas comerciais (NFC), número de folhas totais (NFT), número de brotações (NB). \{ ${ }^{* *}$ significant by F test at $1 \%$. Plant height (AP), stem diameter (DC), leaf length (CF), leaf width (LF), number of marketable leaves (NFC), total number of leaves (NFT), number of shoots $(\mathrm{NB})\}$.

resultados evidenciam a variabilidade existente entre os genótipos estudados, demonstrando que o componente de variância genético, confundido com os efeitos permanentes do ambiente, é significativo (Manfio et al., 2011). Os coeficientes de variação obtidos para esses caracteres foram inferiores a $20 \%$. As características comprimento do limbo foliar, número de folhas comerciais e número de brotações apresentaram os maiores valores para o coeficiente de variação, sendo, possivelmente, os mais influenciados pelo ambiente.
As estimativas dos coeficientes de repetibilidade obtidas pelos diferentes procedimentos estatísticos foram próximas entre os métodos estatísticos utilizados (Tabela 1). Observa-se que as estimativas do coeficiente de repetibilidade obtidas pelo método da análise de variância (ANOVA) são sempre iguais ou inferiores às estimativas obtidas pelos métodos multivariados (análise estrutural e componentes principais). Resultados similares foram obtidos por Lopes et al. (2001), Neto et al.(2002), Feijó et al. (2005), Martuscello et al.
(2007), Chia et al. (2009), Neves et al.(2010) e Manfio et al. (2011). No entanto, as diferenças observadas entre as estimativas obtidas pelos diferentes métodos são pequenas, aumentando a confiabilidade das estimativas.

A inferioridade observada pelo método de ANOVA nos trabalhos relatados é decorrente da variância genotípica, utilizada para estimar a repetibilidade, a qual não é somente de origem genética, uma vez que o componente de variância do ambiente permanente entre indivíduos permanece confundido com

Tabela 2. Estimativas da repetibilidade por diferentes métodos para características avaliadas em 27 clones de couve (repeatability estimates using different methods for characteristics evaluated on 27 kale clones). Diamantina, UFVJM, 2012.

\begin{tabular}{|c|c|c|c|c|c|c|}
\hline \multirow{2}{*}{ Caráter } & & \multirow{2}{*}{ ANOVA } & \multicolumn{2}{|c|}{ Componentes principais } & \multicolumn{2}{|c|}{ Análise estrutural } \\
\hline & & & (Co-variância) & (Correlação) & (Co-variância) & (Correlação) \\
\hline \multirow{2}{*}{ AP } & Coef. & 0,944 & 0,981 & 0,981 & 0,981 & 0,944 \\
\hline & $\mathrm{R}^{2}(\%)$ & 99,407 & 99,807 & 99,810 & 99,810 & 99,407 \\
\hline \multirow{2}{*}{$\mathrm{DC}$} & Coef. & 0,900 & 0,975 & 0,975 & 0,975 & 0,900 \\
\hline & $\mathrm{R}^{2}(\%)$ & 98,904 & 99,747 & 99,739 & 99,739 & 98,904 \\
\hline \multirow{2}{*}{$\mathrm{CF}$} & Coef. & 0,830 & 0,862 & 0,914 & 0,907 & 0,830 \\
\hline & $\mathrm{R}^{2}(\%)$ & 97,990 & 98,420 & 99,072 & 98,987 & 97,990 \\
\hline \multirow{2}{*}{ LF } & Coef. & 0,915 & 0,945 & 0,950 & 0,950 & 0,915 \\
\hline & $\mathrm{R}^{2}(\%)$ & 99,081 & 99,423 & 99,475 & 99,473 & 99,081 \\
\hline \multirow{2}{*}{ NFC } & Coef. & 0,833 & 0,939 & 0,948 & 0,948 & 0,833 \\
\hline & $\mathrm{R}^{2}(\%)$ & 98,031 & 99,353 & 99,453 & 99,449 & 98,031 \\
\hline \multirow{2}{*}{ NFT } & Coef. & 0,978 & 0,983 & 0,983 & 0,983 & 0,978 \\
\hline & $\mathrm{R}^{2}(\%)$ & 99,778 & 99,826 & 99,825 & 99,825 & 99,778 \\
\hline \multirow{2}{*}{ NB } & Coef. & 0,843 & 0,881 & 0,882 & 0,881 & 0,843 \\
\hline & $\mathrm{R}^{2}(\%)$ & 98,176 & 98,671 & 98,674 & 98,671 & 98,176 \\
\hline
\end{tabular}

Altura da planta (AP), diâmetro do caule (DC), comprimento da folha (CF), largura da folha (LF), número de folhas comerciais (NFC), número de folhas totais (NFT), número de brotações (NB). \{ plant height (AP), stem diameter (DC), leaf length (CF), leaf width (LF), number of commercial leaves (NFC), total number of leaves (NFT), number of shoots (NB)\}. 
Tabela 3. Estimativa do número mínimo de medições por diferentes métodos para características avaliadas em 27 clones de couve (estimate of the minimum number of measurements using different methods for the evaluated characteristics on 27 kale clones). Diamantina, UFVJM, 2012.

\begin{tabular}{|c|c|c|c|c|c|}
\hline \multirow{2}{*}{ Caráter } & \multirow{2}{*}{$\mathbf{R}^{2}$} & \multirow{2}{*}{ ANOVA } & \multicolumn{2}{|c|}{ Componentes principais } & \multirow{2}{*}{$\begin{array}{c}\text { Análise } \\
\text { estrutural }\end{array}$} \\
\hline & & & (Co-variância) & (Correlação) & \\
\hline \multirow{5}{*}{ AP } & 0,80 & 0,239 & 0,077 & 0,076 & 0,076 \\
\hline & 0,85 & 0,338 & 0,110 & 0,108 & 0,108 \\
\hline & 0,90 & 0,537 & 0,174 & 0,171 & 0,172 \\
\hline & 0,95 & 1,134 & 0,368 & 0,362 & 0,362 \\
\hline & 0,99 & 5,909 & 1,915 & 1,885 & 1,887 \\
\hline \multirow{5}{*}{ DC } & 0,80 & 0,443 & 0,101 & 0,105 & 0,105 \\
\hline & 0,85 & 0,628 & 0,144 & 0,148 & 0,149 \\
\hline & 0,90 & 0,997 & 0,228 & 0,236 & 0,236 \\
\hline & 0,95 & 2,105 & 0,481 & 0,498 & 0,498 \\
\hline & 0,99 & 10,967 & 2,508 & 2,594 & 2,596 \\
\hline \multirow{5}{*}{$\mathrm{CF}$} & 0,80 & 0,821 & 0,642 & 0,375 & 0,409 \\
\hline & 0,85 & 1,162 & 0,910 & 0,531 & 0,580 \\
\hline & 0,90 & 1,846 & 1,445 & 0,843 & 0,921 \\
\hline & 0,95 & 3,898 & 3,051 & 1,779 & 1,944 \\
\hline & 0,99 & 20,309 & 15,899 & 9,270 & 10,128 \\
\hline \multirow{5}{*}{ LF } & 0,80 & 0,371 & 0,232 & 0,211 & 0,212 \\
\hline & 0,85 & 0,526 & 0,329 & 0,299 & 0,300 \\
\hline & 0,90 & 0,835 & 0,522 & 0,475 & 0,477 \\
\hline & 0,95 & 1,763 & 1,103 & 1,003 & 1,007 \\
\hline & 0,99 & 9,184 & 5,747 & 5,225 & 5,248 \\
\hline \multirow{5}{*}{ NFC } & 0,80 & 0,803 & 0,260 & 0,220 & 0,222 \\
\hline & 0,85 & 1,138 & 0,369 & 0,312 & 0,314 \\
\hline & 0,90 & 1,808 & 0,586 & 0,495 & 0,499 \\
\hline & 0,95 & 3,816 & 1,237 & 1,045 & 1,053 \\
\hline & 0,99 & 19,886 & 6,447 & 5,444 & 5,485 \\
\hline \multirow{5}{*}{ NFT } & 0,80 & 0,089 & 0,070 & 0,070 & 0,070 \\
\hline & 0,85 & 0,126 & 0,099 & 0,099 & 0,099 \\
\hline & 0,90 & 0,200 & 0,157 & 0,158 & 0,158 \\
\hline & 0,95 & 0,422 & 0,332 & 0,333 & 0,333 \\
\hline & 0,99 & 2,201 & 1,729 & 1,733 & 1,734 \\
\hline \multirow{5}{*}{ NB } & 0,80 & 0,743 & 0,539 & 0,537 & 0,539 \\
\hline & 0,85 & 1,053 & 0,763 & 0,761 & 0,763 \\
\hline & 0,90 & 1,672 & 1,212 & 1,209 & 1,212 \\
\hline & 0,95 & 3,530 & 2,559 & 2,553 & 2,559 \\
\hline & 0,99 & 18,394 & 13,332 & 13,301 & 13,336 \\
\hline
\end{tabular}

Altura da planta (AP), diâmetro do caule (DC), comprimento da folha (CF), largura da folha (LF), número de folhas comerciais (NFC), número de folhas totais (NFT), número de brotações (NB). \{ plant height (AP), stem diameter (DC), leaf length (CF), leaf width (LF), number of commercial paper (NFC), total number of leaves (NFT), number of shoots (NB) $\}$.

a variância genotípica (Costa, 2003). $\mathrm{O}$ método dos componentes principais permite isolar o efeito da alternância, o que não ocorre na análise de variância, ficando esse componente incluído no erro experimental (Manfio et al., 2011).

O comprimento do limbo foliar, número de folhas comerciais e o número de brotações foram as únicas características que apresentaram estimativas do coefi- ciente de repetibilidade inferior a 0,90 segundo o método da ANOVA $(0,800$; 0,833 e 0,843 ; respectivamente). As demais características apresentaram altos valores para este coeficiente, indicando pouco acréscimo na precisão experimental em função do aumento do número de medições. De acordo com Padilha et al. (2003), valor acima de 0,5 para o coeficiente de repetibilidade indica que existe confiabilidade significativa para os números de medições necessárias.

As estimativas dos coeficientes de repetibilidade, expressam o valor máximo que a herdabilidade no sentido amplo pode atingir (Cruz et al., 2012), tornando-se importantes para programas de melhoramento desta cultura. Segundo Costa (2003), a diferença entre a repetibilidade e a herdabilidade se deve ao fato de que a variância genotípica utilizada para estimar a repetibilidade não é somente de origem genética, uma vez que o componente de variância do ambiente permanente entre indivíduos permanece confundido com esta. De acordo com os mesmos autores, a repetibilidade aproxima-se da herdabilidade à medida que a variância proporcionada pelos efeitos permanentes do ambiente é minimizada.

Todas as estimativas dos coeficientes de determinação, obtidas para as características pelos quatro métodos foram superiores a 97,9\% (Tabela 2), demonstrando que a avaliação das características pode ser realizada com alta confiabilidade. Segundo Cardoso (2006), estimativas do coeficiente de determinação acima de $80 \%$ podem ser consideradas razoáveis.

A partir dos métodos multivariados verificou-se que uma única medição é suficiente para a avaliação de todas as características com coeficiente de determinação de $95 \%$ (Tabela 3), exceto para o comprimento do limbo foliar e o número de brotações, quando são necessárias três medições. Pelo método da ANOVA, verificou-se a necessidade de um maior número de avaliações, contudo, neste método o coeficiente de repetibilidade possui menor confiabilidade, conforme já comentado anteriormente.

O maior número de medições necessárias para o comprimento do limbo foliar e para o número de brotações pode 
ser devido à maior interação entre os genótipos e o ambiente temporário nestas características. Outra justificativa para a necessidade de maior número de medições, segundo Cruz et al. (2012), é a possibilidade do caráter ser regulado por conjuntos gênicos diferentes, podendo eles estarem mais ou menos ativos em função do estado de desenvolvimento do indivíduo.

Logo, com apenas três colheitas é possível comparar clones de couve, evitando a perda de tempo, avaliando-se os genótipos além do necessário ou avaliando por um período muito pequeno, o que poderia levar a erros na identificação dos genótipos superiores.

\section{AGRADECIMENTOS}

À Fundação de Amparo à Pesquisa do estado de Minas Gerais (FAPEMIG), ao Conselho Nacional de Desenvolvimento Científico e Tecnológico (CNPq) e à Coordenação de Aperfeiçoamento de Pessoal de Nível Superior (CAPES) pela concessão de bolsas de estudos e recursos financeiros para o desenvolvimento do projeto.

\section{REFERÊNCIAS}

AZEVEDO AM; ANDRADE JÚNIOR VC;
PEDROSA CE; FERNANDES JSC; VALADARES NR; FERREIRA MRA; MARTINS RAV. 2012. Desempenho agronômico e variabilidade genética em genótipos de couve. Pesquisa Agropecuária Brasileira 47: 1751-1758.

BALKAYAA; YANMAZ R. 2005. Promising kale (Brassica oleracea var. acephala) populations from Black Sea region, Turkey. New Zealand Journal of Crop and Horticultural Science 33: 1-7.

CARDOSO AII. 2006. Número mínimo de colheitas em pepino híbrido estimado por meio do coeficiente de repetibilidade. Bragantia 65: 591-595.

CHIA GS; LOPES R; CUNHA RNV; ROCHA RNC; LOPES MTG. 2009. Repetibilidade da produção de cachos de híbridos interespecíficos entre o caiaué e o dendezeiro. Acta Amazônica 39: 249-254.

COSTA JG. 2003. Estimativas de repetibilidade de alguns caracteres de produção em mangueira. Ciência Rural 33: 263-266.

CRUZ CD. 2013. GENES - a software package for analysis in experimental statistics and quantitative genetics. Acta Scientiarum Agronomy 35: 271-276.

CRUZ CD; REGAZZI AJ; CARNEIRO PCS. 2012. Modelos Biométricos Aplicados ao Melhoramento Genético. Viçosa: UFV. 514p.

FEIJÓ S; OLIVEIRA SJR; STORCK L; LÚCIO AD; DAMO HP; MARTINI LFD. 2005. Repetibilidade da produção de frutos de abobrinha italiana. Revista Brasileira de Agrociência 11: 39-43.

FILGUEIRA FAR. 2008. Novo manual de olericultura: Agrotecnologia moderna na produção e comercialização de hortaliças. Viçosa: UFV. 402p.
LOPES R; BRUCKNER CH; CRUZ CD; LOPES MTG; FREITAS GB. 2001. Repetibilidade de características do fruto de aceroleira. Pesquisa Agropecuária Brasileira 36: 507-513.

MANFIO CE; MOTOIKE SY; SANTOS CEM; PIMENTEL LD; QUEIROZ V; SATO AY. 2011. Repetibilidade em características biométricas do fruto de macaúba. Ciência Rural 41: 70-76.

MARTUSCELLO JA; JANK L; FONSECA DM; CRUZ CD; CUNHA DNFV. 2007. Repetibilidade de caracteres agronômicos em Panicum maximum Jacq. Revista Brasileira de Zootecnia 36: 1975-1981.

MORENO DA; CARVAJAL M; LOPEZBERENGUER C; GARCIA-VIGUERA C. 2006. Chemical and biological characterization of nutraceutical compounds of broccoli. Journal Pharm Biomed Anal 41: 1508-1522.

NEVES LG; BRUCKNER CH; CRUZ CD; BARELLI MAA. 2010. Avaliação da repetibilidade no melhoramento de famílias de maracujazeiro. Revista Ceres 57: 480-485.

NETO JTF; YOKOMIZO G; BIANCHETTI A. 2002. Coeficientes de repetibilidade genética de caracteres em Pupunheira. Revista Brasileira de Fruticultura 24: 731-733.

NOVO MCSS; PRELA-PANTANO A; TRANI PE; BLAT SF. 2010. Desenvolvimento e produção de genótipos de couve manteiga. Horticultura Brasileira 28: 321-325.

PADILHA NCC; OLIVEIRA MSP; MOTA MGC. 2003. Estimativa da repetibilidade em caracteres morfológicos e de produção de palmito em pupunheira (Bactris gasipaes Kunth). Revista Árvore 27: 435-442.

VILAR M; CARTEA ME; PADILLA G. 2008. The potential of kales as a promising vegetable crop. Euphytica 159: 153-165. 